\title{
CARACTERIZACIÓN BIOGEOGRÁFICA Y CONSERVACIÓN DE LAS LOMAS DE QUEBRADA DE BURROS, TACNA
}

\author{
RESULTADOS PRELIMINARES
}

José Pizarro Neyra ${ }^{1}$

\author{
R E S U M E N
}

\begin{abstract}
Se presentan datos sobre la flora y fauna de la zona denominada "Quebrada de burros" (1803'00" Latitud Sur, 7049'01" Longitud Oeste) tomados en sucesivos muestreos realizados entre noviembre de 1994 y diciembre de 1995. También se consigna información general sobre características climatológicas y geomorfológicas de la quebrada. Todo lo anterior sirve para demostrar que el lugar es único, desde el punto de visla biogeográfico, en la región.
\end{abstract}

\section{INTRODUCIÓN}

A lo largo de la costa del Perú y Norte de Chile existen diversas quebradas secas que reciben aguas de escorrentía subterránea ó superficial proveniente de la vertiente occidental de los Andes, en los meses de lluvia. Pulgar (1970), define a la vegetación de lomas como : "vegetación espontánea y efímera, que comienza a fines de mayo y dura más o menos hasta fines de octubre". Para Pulgar, Ias Iomas ocupan la región "Chala" con un intervalo altitudinal de 0 a 700 metros sobre el nivel del mar (msnm). Koepcke (citado por ONERN, 1986), clasifica a las lomas en seis tipos, una de los cuales son las lomas de cactus.

Aparentemente la "Quebrada de burros" (en adelante quebrada-1), tiene todas las características de una loma de cactus. Es, hasta el momento, la única biocenosis de su tipo en Tacna que conocemos.

Es preciso reconocer biogeográficamente a quebrada-1 para definir el actual estado de conservación del lugar, el cual viene siendo explotado y deteriorado. El presente trabajo pretende sistematizar toda la información obtenida sobre la zona con fines de caracterización biogeográfica e interpretación ambiental.

1. Director CEIMA, UNJBG.

\section{MATERIALES Y MÉTODOS}

\subsection{MATERIALES}

\section{- Logística}

Se emplearon los siguientes equipos de campo : binoculares marca sotem $8 \times 40$, cámara fotográfica semi-profesional marca zenith, cámara de video V-8 marca sony, regla y wincha de medición, termómetro de escala amplia. La movilidad fue proporcionada en varias ocasiones por la Universidad Nacional de Tacna.

\section{- El ambiente natural}

Quebrada-1 es una quebrada natural de laderas rocosas ubicada en $18^{\circ} 03^{\prime} 00^{\prime \prime}$ Latitud Sur, $70^{\circ} 49^{\prime} 01^{\prime \prime}$ Longitud Oeste. Se encuentra bajo la jurisdicción de la Provincia Jorge Basadre, Subregión Tacna.

\section{- Metodología}

Se usaron las descripciones de Aragón (1982), Ostolaza (1987), Flores (1987), Mostacero y Mejía (1992) y Zegarra (1994) para caracterizar especies botánicas, en especial cactáceas.

Para caracterización de vertebrados, se emplearon las guias de campo de Araya \& Millie (1986), Fjeldsa y Krabe (1990). La artropofauna según diversos autores.

La ubicación y las características morfológicas del terreno se contrastaron con 
ejemplares de la carta geográfica nacional (1964), cuadrángulo La Yarada. Para la medición de parámetros climáticos (humedad relativa y temperatura ambiente) se emplearon termómetros de escala amplia, tanto de medida directa como en mediciones de temperatura de bulbo húmedo.

\section{RESULTADOS}

\section{a) Inventario biológico}

El inventario biológico tentativo realizado hasta diciembre de 1995 está resumido en el siguiente cuadro:

CUADRO N 01: Flora y Fauna de Quebrada de burros.

\begin{tabular}{|c|c|}
\hline FLORA & FAUNA \\
\hline DIVISION CHLOROPHYTA & CLASE INSECTA \\
\hline Chrococcus sp. & $\begin{array}{l}\text { Gryllus sp. } \\
\text { Tipuia sp } \\
\text { Pepsis sp. }\end{array}$ \\
\hline Enteromorpha sp. & $\begin{array}{l}\text { Escarabajo del gènero Hidrophyllidae } \\
\text { Escarabajo del género Cordibates. } \\
\text { Escarabajo del género Phaleria } \\
\text { Odonatos de las Familias Zigoptera y }\end{array}$ \\
\hline DIVISIONN GYMNOSPERMOPHYTA & $\begin{array}{l}\text { Anisoptera. } \\
\text { Himenóptero no identificado } \\
\text { Termitas del Orden Isoptera. }\end{array}$ \\
\hline Distichlis spicata & $\begin{array}{l}\text { Dipteros de la Familia Simulidae. } \\
\text { Dipteros de la Familia Tabanidae }\end{array}$ \\
\hline Tillandsia wedermani & CLASE ARACHNIDA \\
\hline $\begin{array}{l}\text { Nolana spp. } \\
\text { Haagocereus spp. }\end{array}$ & $\begin{array}{l}\text { Hadruroides lunatus } \\
\text { Argiope argentata } \\
\text { Tomis spp. } \\
\text { Otros aráenidos no identificados } \\
\text { Cienpies del Orden Scolopendromorpha }\end{array}$ \\
\hline Neoraimondea spp. & OTROS INVERTEBRADOS : \\
\hline Weberbacreus spp. & $\begin{array}{l}\text { Gasterópodos no identificados. } \\
\text { Crustáceos de la Familia Porciollionidae. } \\
\text { Clase Amphibia }\end{array}$ \\
\hline Croton spp & $\begin{array}{l}\text { Buffo SPP } \\
\text { CLASE REPTILIA }\end{array}$ \\
\hline $\begin{array}{l}\text { Lycopersicon chilense } \\
\text { Grindellia glutinosa }\end{array}$ & $\begin{array}{l}\text { Microlophus spp. } \\
\text { Phyllodactylus gerrophygus }\end{array}$ \\
\hline Gindellia glutinosa & Ofidıo de Familia Colubridae \\
\hline Pluchea chingoyo & Clase Aves \\
\hline Ipomoea spp & $\begin{array}{l}\text { Phalcobaenus ssp. } \cdot \\
\text { Cathartes aura jota } \\
\text { Geositta ssp. } \\
\text { Notiochelidon cyanoleuca } \\
\text { Columba fasciata }\end{array}$ \\
\hline Bacopa monnieri & Clase Mammalia \\
\hline Cynodon datylon & $\begin{array}{l}\text { Pseudalopex } 5 s p . " \\
\text { Equinus asinus } " * \\
\text { Lama guanicoe } * \\
\text { Hipocamelus antisensis * }\end{array}$ \\
\hline Hidrocotyle bonaeriensis & Bos taurus \\
\hline & Ratón del Orden Rodentia $\cdots$ \\
\hline
\end{tabular}

- Caracterizados en base a restos de osamenta.

* Observados desde lejos.

** Caracterizados en base a restos de fecas.

\section{b) Datos climatológicos}

Los resultados de medición de temperatura ambiental y humedad relativa fueron los siguientes:

- Temperatura (13 Agosto 1995)

$\left(8 \mathrm{am}=21^{\circ} \mathrm{C} ; 5 \mathrm{pm}=19^{\circ} \mathrm{C}\right)$

- Humedad relativa (13 Agosto 1995)

$$
(8 \mathrm{am}=69 \% ; 5 \mathrm{pm}=71 \%)
$$

\section{c) Morfología del terreno}

La quebrada sufre una bifurcación en dos ramales a una altura de 250 metros de altura. Uno de ellos se dirige hacia el Morro Sama en dirección Nor Oeste y el otro sigue elevándose hacia Cerro Batancito en dirección Nor Este. Es la única quebrada con cobertura vegetal entre muchos kilómetros cuadrados de desierto.

En el contexto de quebrada-1 se distinguen tres tipos de lomas:

- Lomas pedregosas: recorren la quebrada por aproximadamente $3 \mathrm{Km}$ desde la zona supralitoral, en el Océano Pacífico, hasta los $500 \mathrm{msnm}$. La capa superficial del suelo es escasa : en el centro de la quebrada es de tipo aluvial y pedregoso en la parte más cercana al mar (situada entre Punta Gallinazo y Punta Mesa).

- Lomas arenosas hiperáridas: se encuentran superando los $500 \mathrm{msnm}$, en la cabecera de la quebrada, donde parece terminar el nivel del ojo de agua. El suelo es de contextura francoarenosa y cerca a las laderas es de origen aluvial.

- Lomas arenosas: se encuentran a los lados de la quebrada, superando las laderas rocosas. EI origen del suelo es eólico, con dunas en la parte superior de la quebrada.

\section{d) Fitosociologia}

Se han determinado las siguientes asociaciones vegetales:

- Asociación Higrófita : con presencia de Bacopa monnieri cerca al agua proveniente de una fuente al final de la quebrada. Otras plantas son Cynodon dactylon, Distichlis spicata, Ipomoea sp., Hidrocotyle bonaeriensis, Acacia sp. y una compuesta de cojin no identificada cerca al ojo de agua. La flora criptogámica resaltante es Enteromorpha sp.

- Asociación Hiperxerófita : Grindellia glutinosa y Tillandsia $s p$. en lomas arenosas. 
- Asociación de cactáceas columnares : asociación entre Neoraimondea spp. aff. arequipensis y una densidad baja de otras fanerógamas como Haagocereus sp., Grindellia glutinosa, Lycopersicon chilense, Weberbacereus spp. sobre las laderas rocosas de la quebrada hasta los $500 \mathrm{msnm}$.

- Asociación de piso de quebrada : compuesta por el grueso de fanerógamas, siendo predominante Grindellia glutinosa y Distichlis spicata.

\section{e) Poblaciones animales predominantes}

Entre los vertebrados, las únicas poblaciones numerosas son las del Furnárido del Género Geositta. Estas aves ocupan cuevas altas en la quebrada ó cerca a las fuentes de agua. Son también numerosas las lagartijas del Género Microlophus. Los sapos del género Buffo son abundantes sólo en la cercania del ojo de agua.

Entre los invertebrados se distinguen comunidades, como la formada por Cordibates spp, y termitas, alrededor de cactus del Género Neoraimondea que se encuentran secos; Argiope argentata sobre arbustos de Grindellia glutinosa; Simúlidos y Tipúlidos cerca a los abrevaderos, donde habita Buffo spp. ; Phaleria sp. entre restos de excrementos de vertebrados.

\section{f) Estado de conservación actual}

La última visita a la zona, realizada el 16 de diciembre, revela que la destrucción de la quebrada acontece hasta la bifurcación, afectando drásticamente la cobertura vegetal, obstrucción y desecamiento de riachuelos y pozos, destrucción de laderas rocosas y ahuyentamiento de especies animales por el ruido de las explosiones de dinamita y en general, una grave pérdida de la biodiversidad en la zona.

\section{DISCUSIÓN DE RESULTADOS}

Los datos climáticos preliminares revelan una humedad relativa media-alta debido a la presencia de neblinas costeras. Pinche (1993), informó que en las cercanías de Cerro Meca y Cerro Batancito se podian recoger hasta 10 litros de agua de niebla al dia con colectores de 1 metro cuadrado de superficie efectiva durante los meses de niebla. Esto dá una idea de lo importante que son las neblinas costeras para existencia de la vida en quebrada-1 y las posibilidades que tiene para su conservación. Según
Schemenauer y Cereceda (1994), de 22 paises estudiados en el mundo, los mejores resultados obtenidos en experiencias de recojo de agua de nieblas se han dado en Perú y el Sultanato de Omán, con promedios de 9-10 litros/dia.

Los cactus representan las poblaciones más numerosas. Algunas de ellas (como las del género Haagocereus) han sido recomendadas por Ostolaza (1987) para ser incluidas en el apéndice I de CITES. Se dan similitudes entre cactáceas que se distribuyen altitudinalmente entre 0 y 200 msnm en Arequipa. Villasante y col. (1995), mencionan para ésa zona en Arequipa a los Géneros Haagocereus y Neoraimondea, las cuales están presentes en quebrada-1. La distribución de Neoraimondea ap. en pisos tan bajos queda comprobada, y corrige las afirmaciones de Zegarra (1994), en cuanto a los cactus que habitan el piso de cactáceas columnares entre 800-900 hasta $2000-2600$ metros de altura en la provincia de Jorge Basadre.

Para las lomas de Tacahuay, ubicadas al norte de Ite, entre los 150 y 900 msnm, sólo hay similitud en la composición florística en cuanto a arbustos como Croton sp. y Grindellia glutinosa.

Para efectos de comparación con otras lomas de costa, se ha elaborado el cuadro $\mathrm{N}^{\circ} 2$.

CUADRO Nº2 : Distribución de Cactáceas en pisos bajos de distintas lomas costeras del Sur del Perú.

\begin{tabular}{|l|l|}
\hline \multicolumn{1}{|c|}{ LOMA } & \multicolumn{1}{c|}{ CACTACEAS } \\
\hline $\begin{array}{l}\text { Tacahuay (Tacna-Moquegua) } \\
\text { Según Luyo, 1995. } \\
\text { Sama (Tacna) Según Zegarra, } \\
1994 .\end{array}$ & Corryocactus sp. \\
$\begin{array}{l}\text { Quebrada de burros (Tacna) } \\
\text { Este trabajo. }\end{array}$ & $\begin{array}{l}\text { Neoraimondea spp., } \\
\text { Haagocereus spp., } \\
\text { Weberbacereus sp. }\end{array}$ \\
$\begin{array}{l}\text { Arequipa } \\
\text { Villasante y col. } 1995\end{array}$ & $\begin{array}{l}\text { Géneros: Opuntia, Islaya, } \\
\text { Haagocereus, Neoraimondea, } \\
\text { Cereus, Borziocactus. }\end{array}$ \\
\hline
\end{tabular}

Las especies leñosas están casi ausentes en quebrada-1. Sólo encontramos a la leguminosa Acacia spp. "Yaro" a un lado de la acequia que discurre hacia el mar en forma aislada y escasa. Esto contrasta con la composición florística de Tacahuay, en la que predomina Caesalpinea spinosa (Luyo, 1995) ó de los oasis y quebradas del Norte de Chile, en las que crecen algarrobos, Tamarugo y Chañar (Hernández, 1973). 
Las especies introducidas son pocas pero tienen presencia prevalente en la zona. Las más numerosas son : Croton sp., Cynodon dactylon, Equs asinus y Bos taurus. Quebrada-1 parece haber mantenido una población numerosa de Bos taurus en el pasado, a decir por abrevaderos y corralones para ganado encontrados alli, la cual no habria excedido la capacidad de carga de la quebrada.

Desde el punto de vista biogeográfico, Cabrera y Willink (1980), incluirian la quebrada de burros en el Distrito del Desierto Costero del Pacífico, donde hay vegetación permanente en lugares cerca al rio y lugares cerca al mar. Los mismos autores incluyen una fotografia en su trabajo donde muestran a un ejemplar de Neoraimondea macrostibas para la costa central del Perú.

Las comunidades de cactáceas son muy importantes desde el punto de vista biogeográfico. Marin (1995), sugiere que en el Dominio desértico sudamericano, las cactáceas constituyen un territorio fitogeográfico totalmente particular formando un Reino, con más de 30 géneros y 400 especies.

De acuerdo a los indicios de presencia de vertebrados, se puede deducir que hay presencia, por lo menos estacional, de $H$. antisensis y $L$. guanicoe. Ellas, junto de Equinus asinus asilvestrados, constituyen recursos que tienden a desaparecer de la zona debido a presión cinegética. La destrucción del hábitat puede considerarse como una presión adicional sobre las poblaciones de vertebrados silvestres en Tacna. La mayoria de éstos vertebrados son de procedencia andino-patagónica.

Las consideraciones de orden biológico para conservar la quebrada de burros giran en torno a la conservación de la biodiversidad. Esta es definida por la UICN (1991) como: "la variedad total de estirpes genéticas, especies y ecosistemas... debe conservarse como una cuestión de principio, pues todas las especies merecen respeto, independientemente de su utilidad para la humanidad... la diversidad biológica también nos proporciona beneficios económicos y mejora en gran

\section{REFERENCIAS BIBLIOGRÁFICAS}

OSTOLAZA, Carlos. Los cactus de los alrededores de Lima y su consevación. Bol Zonas Aridas (5):7-19. 1988. CIZA. Lima.

ARAGON, Giovanni. Cactáceas de los alrededores de la Ciudad de Arequipa. Bol. de Lima IV(21): 86-94. 1982.

CABRERA. A. y A. WILLINK. 1980. Biogeografía de América Latina. 2da. edición. Sec. Gral. OEA, PRDCT, Washington. medida nuestra calidad de vida". Arias y Torres (1989), afirman que las lomas son importantes centros de diversidad biológica, donde se encuentran muchas especies de plantas cultivadas (como el Tomate por ejemplo). La pérdida de biodiversidad es contraria a los principios del Acuerdo de Biodiversidad, firmado por el Gobierno del Perú en 1992 y ratificado por el $\mathrm{CCD}$ al año siguiente.

Otras consideraciones son de carácter educativo. Las lomas como Quebrada-1, siguiendo un mecanismo de interpretación ambiental, pueden ser objeto de estudio permanente con fines didácticos.

Finalmente, está la alternativa de promoción turistica de los atractivos de la vía costanera, que puede destacar a Quebrada-1 como lugar de deportes de aventura y excursiones a lugares de valor arqueológico los cuales son, según Gordillo (1994): "la bifurcación", "los batanes", "el gentilar" y "la cabecera". El mismo autor sitúa a quebrada-1 dentro de la misma unidad fisiográfica de Morro Sama, lugar que merece ser conservado, según Zegarra (1994), debido a su gran valor ecológico, fitogeográfico y florístico.

\section{A MODO DE CONCLUSIÓN}

"Quebrada de burros" comprende un conjunto de espacios vitales que albergan especies residentes y estacionales caracterizados como lomas costeras de cactus ubicadas en la ecoregión del Desierto Costero ó la provincia desértica del Dominio desértico sudamericano. A su vez parece formar parte de la misma unidad fisiográfica que Morro Sama.

Los resultados preliminares del inventario biológico y de la medición de caracteristicas climáticas de la quebrada demuestran que es factible su conservación a través del rescate de especies nativas y el aprovechamiento del agua de nieblas .

Es necesario establecer un plan de ordenamiento territorial de las áreas de influencia de la vía costanera, que considere la variable ambiental para que en la zona las actividades humanas sean sustentables.

ARIAS, C. y J. TORRES. Dinámica de la vegetación de las lomas del Sur del Perú : estacionalidad y productividad primaria. Caso: Lomas de Atiquipa (Arequipa). Bol. Zonas Aridas (6): 55-57. 1989-1990. CIZA, Lima.

SEDAILO-MPI-LABOR. Boletin Gestión Ambiental. No.2, noviembre 1994. Ed. Labor. Ilo.

AGUILAR, Pedro. Antrópodos del Tillandsial de Punta Hermosa. Rev. Per. Ent. XX(1): 88-92. Junio 1977. 
ARAYA. B. y G. MILLIE. 1986. Guia de campo de las aves de Chile. Ed. Universitaria, Santiago.

MARIN, Felipe. Nuevo esquema fitogeográfico del Perú. Libro de resúmenes VI CONABOT : pp.69-70. Cusco, 1995.

VILLASANTE, F.: JIMENEZ, P. y C. TALAVERA. Distribución altitudinal de las cactáceas en Arequipa. Libro de resúmenes VI CONABOT : pp. 64. Cusco, 1995.

ZEGARRA, R. Vegetación desértica perenne del Departamento de Tacna, Estudio Biosistemático y Ecológico. Rev. Nueva Imagen (5): 53-56. Agosto 1994. Tacna.

GORDILLO, Jesús. Informe técnico No. 090-95-UA-INC-T. Subregión de Cultura de Tacna, 1995.

MOSTACERO, J. y F. MEJIA 1993. Taxonomía de fanerógamas peruanas. Ed. ausp. por CONCYTEC.

PIZARRO, J. Observaciones de aves en la costa de Tacna.
Ponencia Jornada Nacional Ornitológica, Lima, Agosto 1995.

UICN-WWF-PNUMA. 1991. Cuidar la Tierra. Estrategia para el Futuro de la Vida. UICN Headquarters, Gland, Suiza.

PINCHE, Cristóbal. Conferencia sobre las perspectivas de utilización de atrapanieblas en la costa de Tacna. Colegio de Ingenieros -Filial Tacna, 1993.

SCHEMENAUER R. y P.CERECEDA Fog collection's role in water planning for developing countries. Natural Resources Forum 18(z): 98. 1994.

PULGAR VIDAL, J. 1970. Geografia del Perú. Ed. Universo, Lima. LUYO, M. 1995. Informe de proyecto de forestación de Quebrada Tacahuay. Labor. Ilo.

HERNÁNDEZ, S. 1973. Geografia de plantas y Animales de Chile. Ed. Universitaria, Santiago.

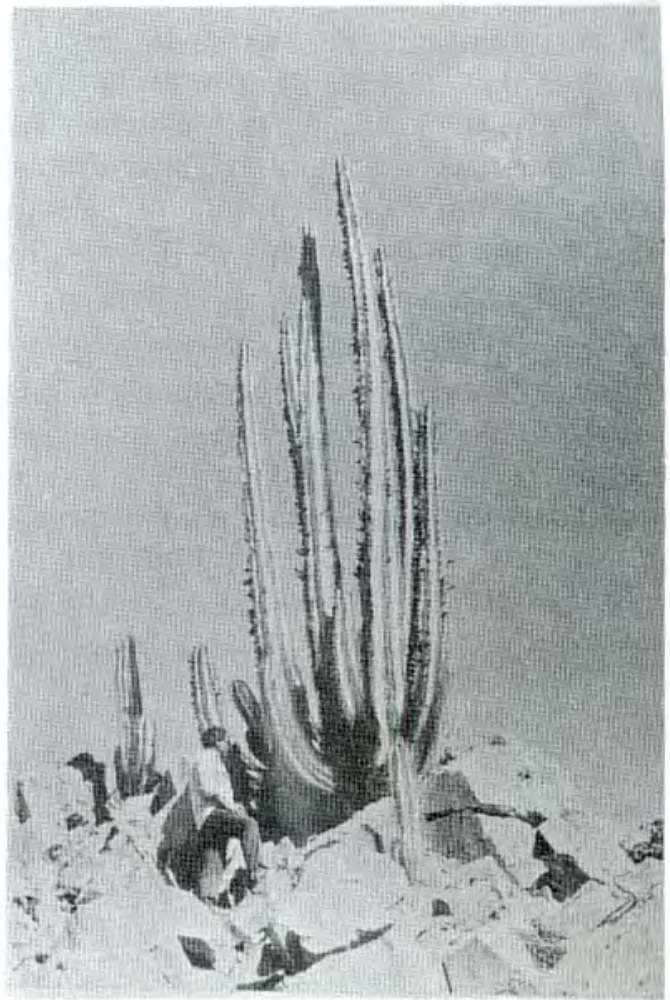

Ejemplar de Neoraimondea sp., Cactus muy común en la zona de Quebrada de Burros, donde forma densas poblaciones, (Foto: G. Gordillo) 